\title{
Mit Übung ist auch Extremsport machbar
}

Sport und Diabetes ist für viele insulinpflichtige Patienten eine schwierige Kombination, die aber in den Griff zu bekommen ist. Die Regeln einer funktionierenden Insulintherapie - Basalrate, Mahlzeiten-Insulin und Berechnung der Broteinheiten müssen stimmen - gelten genauso während des Sports, betonte Heiko Müller, Diabetesberater an der Deutschen Klinik für Diagnostik in Wiesbaden, beim Diabeteskongress in Hamburg.

Zu den größten Ängsten von körperlich aktiven insulinpflichtigen Diabetikern, auch solchen mit Pumpentherapie, zählen Unterzuckerungen beim Sport. Viele Patienten reduzieren deshalb die Dosis des Basalinsulins auf $50 \%$ und mehr am Trainingstag, andere nehmen vor dem Sport eine Extraportion Kohlenhydrate zu sich, ohne dies eigentlich zu wollen. „Die meisten Diabetiker wünschen sich, einmal Sport machen zu können, ohne vorher etwas essen zu müssen“, sagte Müller. Dies ist nach seiner Erfahrung möglich.

Sein Tipp für Patienten mit Pumpentherapie vor einem aeroben Ausdauertraining: nüchtern und ohne Bolus kommen und die Basalrate 1,5 Stunden vor dem Training auf $70 \%$ senken. Auch bei einstündigem Cardio-Training bleibe der Nüchternblutzucker stabil, so die Erfahrung von Müller. Auch vor Ausdauerwettkämpfen funktioniere ein solches Schema: letzter Bolus 4,5 Stunden vor dem Start, Basalrate 1,5 Stunden vorher auf $70 \%$ reduzieren. Der Nüchternblutzucker sollte beim Start möglichst stimmen und während des Wettkampfs müssen natürlich genügend Kohlenhydrate zugeführt werden, sagte Müller, beim Fahrradfahren zum Beispiel rund $30 \mathrm{~g}$ pro Stunde. Die Einstellung der Basalrate bei Pumpenträgern vor dem Wettkampf sei abhängig vom Zeitpunkt des letzten Bolus. Kurz wirksame Analoginsuline wirken nach Erfahrung des Diabetesberaters nicht so kurz wie oft behauptet, sondern mindestens 4 Stunden. Zu berücksichtigen ist vor einem Wettkampf zudem die Adrenalinausschüttung, die zu einer stressinduzierten Insulinresistenz führen könne.

\section{Krafttraining muss kein Problem sein}

Auch Krafttraining ist für insulinpflichtige Diabetiker ohne weiteres möglich, betonte André Pascal Volkmann aus Bremen, Fitnesscoach und Ernährungsberater, u.a. der Fußballprofis von Werder Bremen. Er ist seit seinem sechsten Lebensjahr an Typ1-Diabetes erkrankt und hat trotz regelmäßigen Krafttrainings keine Probleme mit einer guten Blutzuckereinstellung. Zwei- bis dreimal pro Jahr nimmt er sogar an Bodybuilder-Wettkämpfen teil. „Leider gibt es nicht besonders viele Informationen zum Thema 'Krafttraining und Diabetes'“, sagte Volkmann. Manche Ärzte rieten ihren Patienten sogar davon ab.

Zwar fallen die Blutzuckerwerte nach seiner Erfahrung beim Krafttraining relativ wenig ab, aber dennoch hält er Werte von $130 \mathrm{mg} / \mathrm{dl}$ vor Beginn des Trainings für „zu gering“. Auch höhere Insulindosen sollten vor dem Training vermieden werden. Volkmann behandelt seinen Typ-1-Diabetes konventionell und

kommt mit einem einmal am Morgen applizierten Basalinsulin aus. Nach jedem Krafttraining nimmt er 300 g Kohlenhydrate zu sich und braucht für eine gute Blutzuckereinstellung nur 1012 Einheiten Insulin.

Mit der richtigen Ernährung könne auch die Insulintherapie günstig beeinflusst werden, so Volkmann. Empfehlenswert sei ein Low-carb-Frühstück am Morgen, anstelle von Brötchen zum Beispiel ein Rührei oder Pfannkuchen. Dies ermögliche einen geringeren Insulinbedarf bei stabileren Blutzuckerwerten. Auch Eiweißriegel mit ganz wenigen Kohlenhydraten seien für sportliche aktive Menschen sehr zu empfehlen und könnten kurz wirksame Insuline auch mal überflüssig machen. Nicht überschritten werden sollte zum Schutz der Nierenfunktion eine Obergrenze der Proteinzufuhr von 2 Gramm pro kg Körpergewicht.

Extremsportler mit Diabetes und Pumpentherapie setzenmeist auf ganz individuelle Konzepte. Schwierig wird es beim Wassersport, wenn keine wassergeschützte Pumpe verwendet wird. Zwar sei es kein Problem, eine Pumpe über 1-2 Stunden abzunehmen, sagte Dr. Ulrike Becker aus Niederkassel-Rheidt. Die niedergelassene Internistin organisiert regelmäßig Sportseminare für Diabetiker. Allerdings dauert ein Extremsport-Wettkampf häufig länger und viele Sportler ziehen es nach ihrer Erfahrung vor, die Pumpentherapie fortzusetzen und, wenn nötig, wasserdicht zu verpacken.

\section{Mountainbiking, Eisklettern und Laufen}

Ein Beispiel: Katja M., seit 1999 an Typ-1-Diabetes erkrankt, seit 2010 auf einer Insulinpumpentherapie und sehr aktive Sportlerin, u.a. im Mountainbiking, Eisklettern und Wandern. Ihre neue Herausforderung: Teilnahme am „strong run“, einem Hindernislauf über $24 \mathrm{~km}$ mit 34 Hindernissen, z.T. mit Wassergräben. Mit einer rund 4-stündigen Dauer war zu rechnen und auf ihre Pumpe wollte sie nicht verzichten, berichtete Becker. Vorteilhaft war ihr wasserdichtes Modell, infrage kommt aber auch das Verpacken einer Pumpe in einer wasserdichten Hülle wie Aquapac. Dabei ist nach Angaben von Becker unbedingt zu beachten: Im niedrigen Dosisbereich könne die Durchflussrate um bis zu $60 \%$ reduziert sein. Zur Sicherheit sollte bei Extremsport auch ein Messgerät mitgenommen werden, das eventuell ebenfalls wasserdicht verpackt werden müsse. Beckers Appel: Alles beim Sport vorher ausprobieren, damit es im Ernstfall keine Probleme gebe.

Roland Fath

Quelle: Session „Sport und Extremsituationen“ beim Diabeteskongress, 25.5.2017 in Hamburg

Der Diabeteskongress 2018 findet vom 9. bis 12. Mai 2018 in Berlin statt.

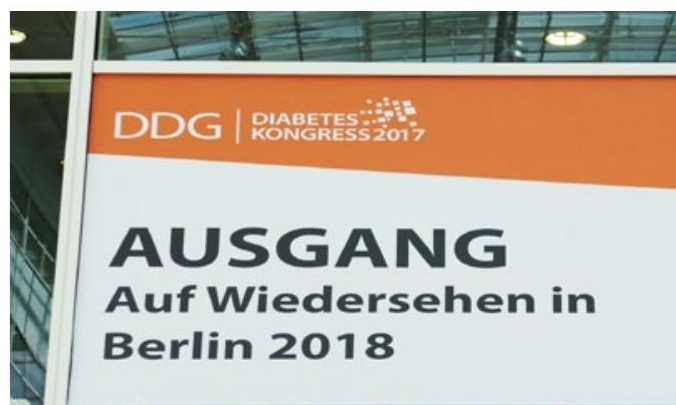

